\title{
PARTICLE NUMBER AND CURRENT IN DIRAC FIELD IN THE CLOSED UNIVERSE
}

\author{
P.R. Dhungel* and U. Khanal* \\ *Central Department of Physics, Tribhuvan University, Kirtipur, Nepal.
}

\begin{abstract}
The behavior of the particle number and particle current of the free Dirac field in the closed FRW universe has been explored. Although the particle number is conserved for the massless fields as expected, it is not for the massive ones. So, there is a finite particle current in any finite sized volume of the universe. Such currents tend to enhance the density contrast over time. It is seen that the momenta of the Dirac particle is quantized in a closed FRW model. The particles distribute themselves in such a way as to resemble that required for the flattened rotation curves of galaxies.
\end{abstract}

Key words: Closed Universe; Dirac field; Comoving particle number density.

\section{INTRODUCTION}

In the Friedmann-Roberston-Walker (FRW) spacetime, the Newman-Penrose formalism [1,2,3] was used to separate the variables and solve the free Dirac field equation in the work of U. Khanal [2]. The FRW metric can be written as

$$
d s^{2}=a^{2}(\eta)\left[d \eta^{2}-d r_{*}^{2}+\frac{\sin ^{2} \sqrt{K} r_{*}}{K}\left(d \theta^{2}+\sin ^{2} \theta d \phi^{2}\right)\right]
$$

where $a$ is the scale factor, $\eta=\int \frac{d t}{a}$ is the conformal time, $r$ * is the tortoise radial coordinate that is related to the usual radial coordinate by $r_{*}=\int d r / f=\frac{2}{\sqrt{K}} \arctan \frac{\sqrt{K} r}{2}$, and $\mathrm{K}=$ $(0,+1$ or -1$)$ for the flat, closed or open universe respectively. The spatial part of the Dirac equation was completely solved. In this paper, we will concentrate on the solution for the closed universe case and discuss the distribution of the Dirac particles and the particle current.

\section{DIRAC EQUATION}

It was shown that the equations governing the free Dirac field reduce to

$\frac{1}{R_{\mp}}\left(\frac{\partial}{\partial r_{*}} \mp \lambda \frac{f}{r}\right) R_{ \pm}=\frac{1}{T_{ \pm}}\left(\frac{\partial}{\partial \eta} \pm i M a\right) T_{\mp}=i k$

where $k=p a$ is the comoving momentum, $l$ in $\lambda=(l+1 / 2)$ is the total angular momentum including the spin as well and hence is a half-integer. The coupled equation (2) can be written as two pairs of decoupled radial and temporal equations for each of different cases flat, closed and open universes. The radial solutions [4] that are regular at the origin, for the closed universe $(K=1)$, are

$R_{ \pm}=A_{ \pm} \sin ^{l+1 \mp \frac{1}{2}}\left(\frac{r_{n}}{2}\right) \cos ^{l+1 \pm \frac{1}{2}}\left(\frac{n_{n}}{2}\right) P_{k-l-1}^{\left(l+\frac{1}{2}+\frac{1}{2}, l+\frac{1}{2} \pm \frac{1}{2}\right)}\left(\cos r_{*}\right) \ldots \ldots$ (3)

where $P_{n}^{(\alpha, \beta)}(x)$ is Jacobi Polynomial [5]. This solution for the closed universe is regular at $r_{*}=\pi$, the maximum value that $r_{*}$ can have, only if $k-l-1=n=$ integer. Although such a quantization of momentum has appeared in the context of closed FRW model, this may be a general rule for all gravitationally bound massive particles.

Writing $x=\cos r_{*}, \alpha=l+\frac{1}{2} \mp \frac{1}{2}, \quad \beta=l+\frac{1}{2} \pm \frac{1}{2}$ and $\mathrm{n}=\mathrm{k}-\mathrm{l}$ -1 , equation (3) can be written as

$R_{ \pm}=A_{ \pm} 2^{-\frac{1}{2}(\alpha+\beta+1)}(1-x)^{\alpha / 2+1 / 4}(1+x)^{\beta / 2+1 / 4} P_{n}^{(\alpha, \beta)}(x) \ldots$

Using the normalization condition: $\int_{0}^{\pi}\left|R_{ \pm}\right|^{2} d r_{*}$, i. e.,

$\left|A_{ \pm}\right|^{2} 2^{-(\alpha+\beta+1)} \int_{-1}^{1}(1-x)^{\alpha}(1+x)^{\beta}\left\{P_{n}^{(\alpha, \beta)}(x)\right\}^{2} d x=1, \ldots$

we get $\left|A_{ \pm}\right|^{2}=\frac{\Gamma(n+1) \Gamma(\alpha+\beta+n+1)(\alpha+\beta+2 n+1)}{\Gamma(\alpha+n+1) \Gamma(\beta+n+1)}$,

or, $\left|A_{ \pm}\right|^{2}=\frac{\Gamma(n+1) \Gamma(2 l+n+2)(2 l+2 n+2)}{\Gamma(l+n+1) \Gamma(l+n+2)}$

\section{PARTICLE NUMBER AND CURRENT}

Since the continuity equation is satisfied by the very structure of the Dirac equation, the particle current is found to be conserved [2]. However, the comoving particle number

Author for Correspondence: U. Khanal, Central Department of Physics, Tribhuvan University, Kirtipur, Nepal. Email: khanalu@yahoo.com. 
is not conserved for massive Dirac field. So, there is a flow of particles into or out of a comoving volume. The comoving particle number density is given by

$n a^{3}=\frac{1}{4 \operatorname{Sin}^{2} r_{*}}\left[\left|Z_{+}\right|^{2}+\left|Z_{-}\right|^{2}\right]\left[\left|Y_{+}\right|^{2}+\left|Y_{-}\right|^{2}\right]$

where $\mathrm{Z}=\mathrm{RT}$ represents the radial and temporal wavefunction, and $\mathrm{Y}$ the angular. Integrating over a solid angle, using the normalization condition $\int\left|Y_{ \pm}\right|^{2} d \Omega=1$, we get

$4 \pi n a^{3}=\frac{1}{2 \operatorname{Sin}^{2} r_{-}}\left[\left|Z_{+}\right|^{2}+\left|Z_{-}\right|^{2}\right]=\frac{1}{2 \operatorname{Sin}^{2} r_{-}}\left[\left|R_{+}\right|^{2}\left|T_{+}\right|^{2}+\left|R_{-}\right|^{2}\left|T_{-}\right|^{2}\right]$

For early universe (at $\eta \rightarrow 0$ ), $\left|T_{ \pm}\right|^{2} \rightarrow \frac{1}{2}$,

$4 \pi \operatorname{Sin}^{2} r_{s} n a^{3}=\frac{1}{4}\left[R_{+}^{2}+R_{-}^{2}\right]$

Using equations (3) or (4), this comoving particle density can be expressed as

\section{$4 \pi \operatorname{Sin}^{2} r_{*} n a^{3}=$}

$\left|A_{ \pm}\right|^{2} 2^{-2 l-4}\left[\left(1-\operatorname{Cos} r_{*}\right)^{l}\left(1+\operatorname{Cos} r_{*}\right)^{l+1}\left\{P_{n}^{(l, l+1)}\left(\operatorname{Cos} r_{*}\right)\right\}^{2}\right.$

$\left.+\left(1-\operatorname{Cos} r_{*}\right)^{l+1}\left(1+\operatorname{Cos} r_{*}\right)^{l}\left\{P_{n}^{(l+1, l)}\left(\operatorname{Cos} r_{*}\right)\right\}^{2}\right]$

Its plots against $r_{*}$ for $l=1 / 2,3 / 2$ and $5 / 2$ for each of $n=0,1$ and 2 have been shown in figures 1a, 1b and 1c respectively. In order to fit all the graphs within the range, they have been appropriately scaled. In fig. 1a, it is seen that the particle density occurs at $r \sim \pi / 2$ for $n=0$. For larger values of $l$, the peaks have become sharper. For $n=1$, there are two maxima, on either sides of $r \sim \pi / 2$. These two overdenisty regions may disjoint over time and could explain the process of fragmentation of large structures. Larger $\mathrm{n}$ appears to produce larger number of such density contrasts, as shown in fig. 1 c, for $n=2$.

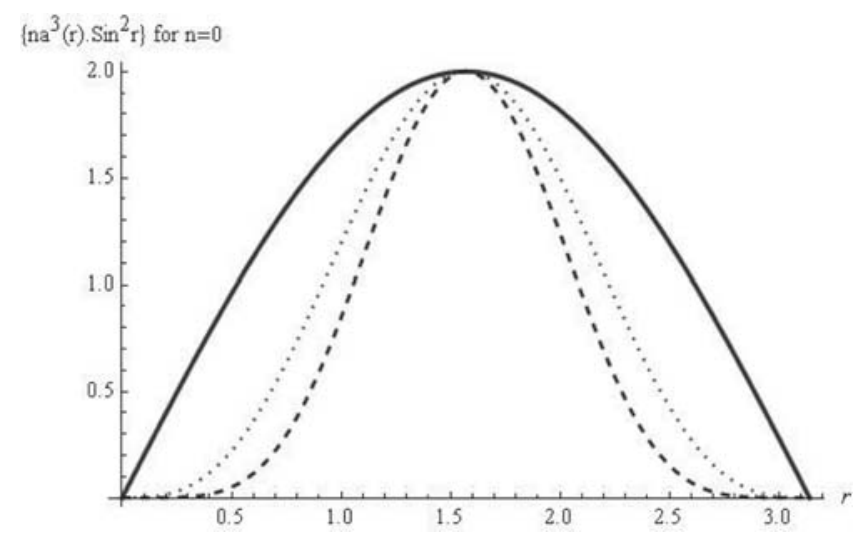

Fig. 1a: The comoving particle number density at a surface of radius $r_{*}$ for $\mathrm{n}=\mathrm{k}-\mathrm{l}-1=0$, i.e., $\mathrm{k}=\mathrm{l}+1$. The solid, dotted and dashed curves are for $l=1 / 2,3 / 2$ and 5/2 respectively. The density has peaked at $r \sim \pi / 2$. For larger values of 1 , the peak has become sharper.

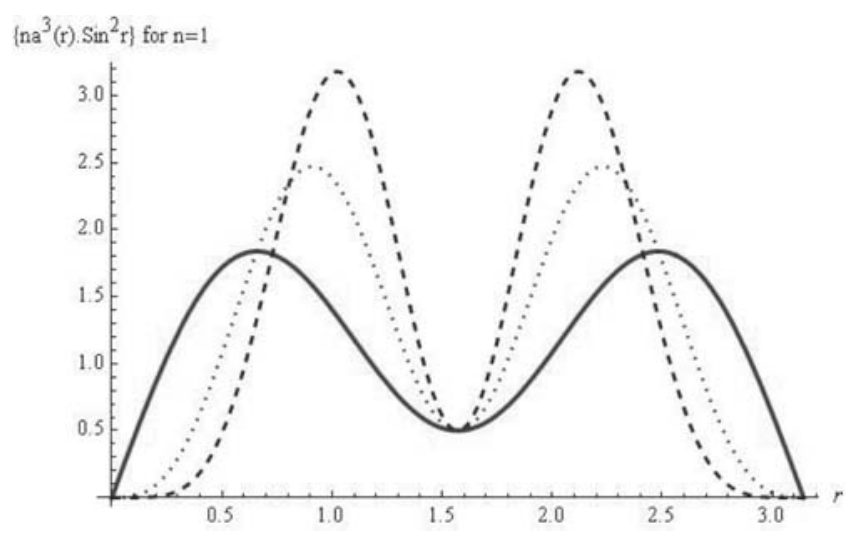

Fig. 1b: The comoving particle number density at a surface of radius $r_{*}$ for $n=k-l-1=1$, i.e., $k=l+2$. The solid, dotted and dashed curves are for $l=1 / 2,3 / 2$ and $5 / 2$ respectively. The peaks indicating overdensity regions have split into two, which may be explained as the process of fragmentation of large structures.

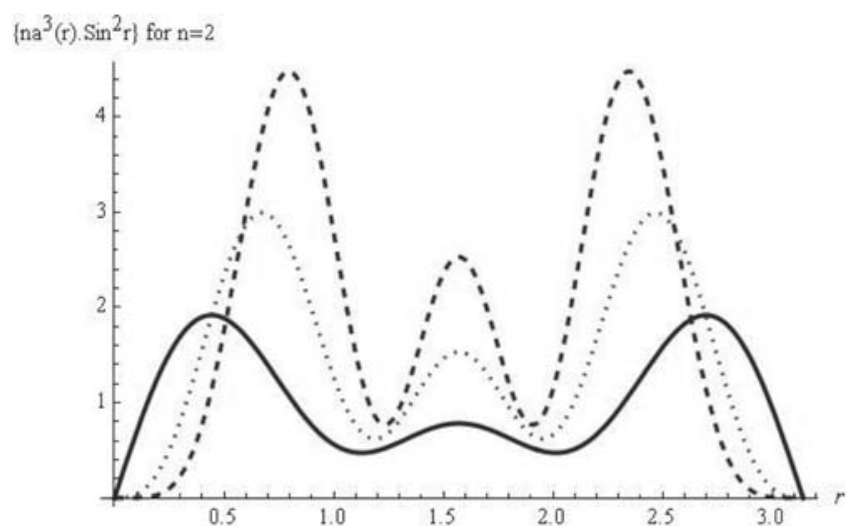

Fig. 1c: The comoving particle number density at a surface of radius $r_{*}$ for $n=k-l-1=2$, i.e., $k=l+3$. The solid, dotted and dashed curves are for $l=1 / 2,3 / 2$ and $5 / 2$ respectively. The peaks indicating overdensity regions have split into three as in $1 \mathrm{~b}$.

Integrating equation (9) over a spatial volume of radius $r_{*}$, we get the total comoving particle number within the volume as

$N a^{3}\left(r_{*}\right)=\int_{0}^{r_{*}<\pi} 4 \pi \operatorname{Sin}^{2} r_{*} n a^{3} d r_{*}=\frac{1}{4} \int_{0}^{r_{0}<\pi}\left[\left|R_{+}\right|^{2}+\left|R_{-}\right|^{2}\right] d r_{*}$

$=\left|A_{ \pm}\right|^{2} 2^{-2 l-4} \int_{0}^{n_{0}}\left[\left(1-\operatorname{Cos} r_{*}\right)^{l}\left(1+\operatorname{Cos} r_{*}\right)^{l+1}\left\{P_{n}^{(l, l+1)}\left(\operatorname{Cos} r_{*}\right)\right\}^{2}\right.$

$\left.+\left(1-\operatorname{Cos} r_{*}\right)^{l+1}\left(1+\operatorname{Cos} r_{*}\right)^{l}\left\{P_{n}^{(l+1, l)}\left(\operatorname{Cos} r_{*}\right)\right\}^{2}\right] d r_{*}$

Its plots against $r_{*}$ for $l=1 / 2,3 / 2$ and $5 / 2$ for each of $\mathrm{n}=0,1$ and 2 have been shown in figures $2 \mathrm{a}, 2 \mathrm{~b}$ and $2 \mathrm{c}$ respectively. Figures 3a, $3 \mathrm{~b}$ and $3 \mathrm{c}$ show the corresponding plots of the comoving particle number per unit $r_{*}$ at a distance $r_{*}$, i.e., $\mathrm{Na}^{3}\left(r_{*}\right) / r_{*}$. It is well known that $\mathrm{MNa}^{3} / \mathrm{r}$ represents the gravitational potential, and is equal to the square of the rotational velocity of particles in Keplerian orbit. Saturation of this quantity at large distance is an important feature of 
the rotation curves of spiral galaxies. Here, it is seen that the Dirac particles distribute themselves in such a density profile that it resembles with the shape of the observed rotation curve of galaxies.
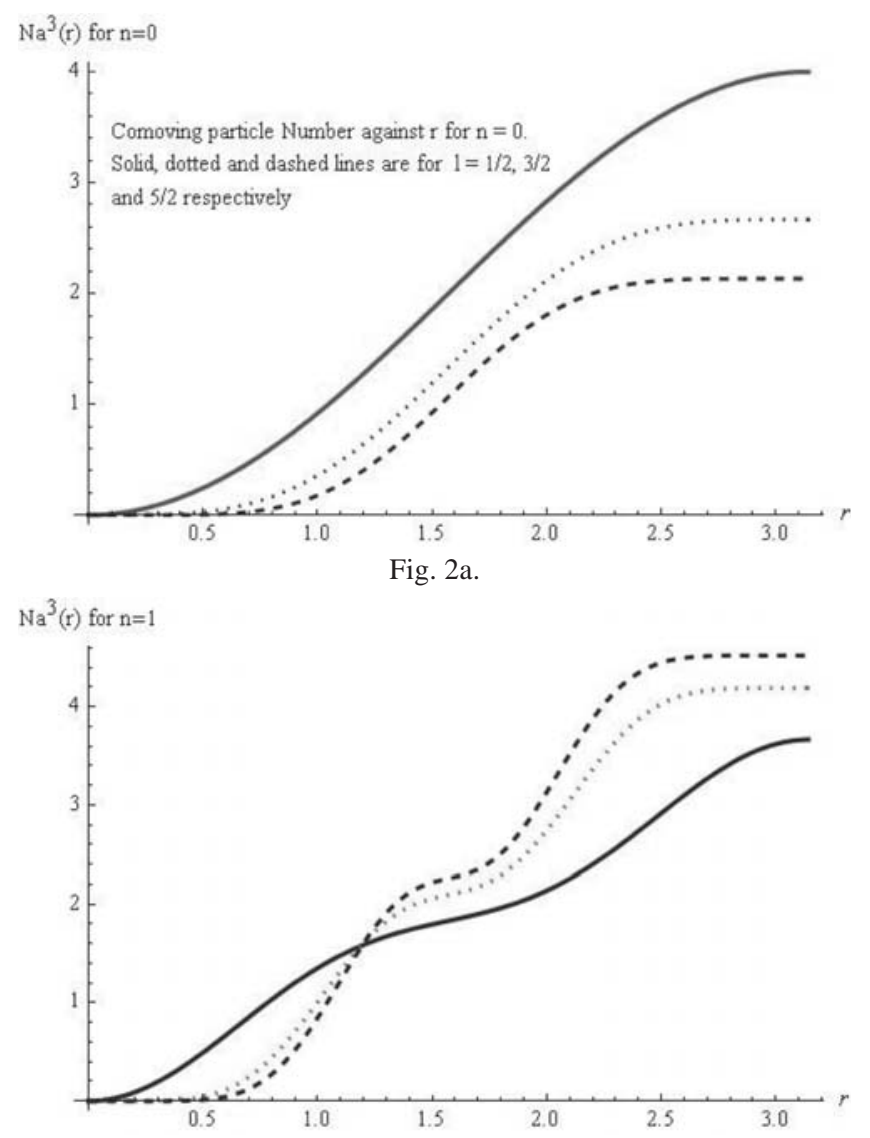

Fig. 2b.

$\mathrm{Na}^{3}(\mathrm{r})$ for $n=3$

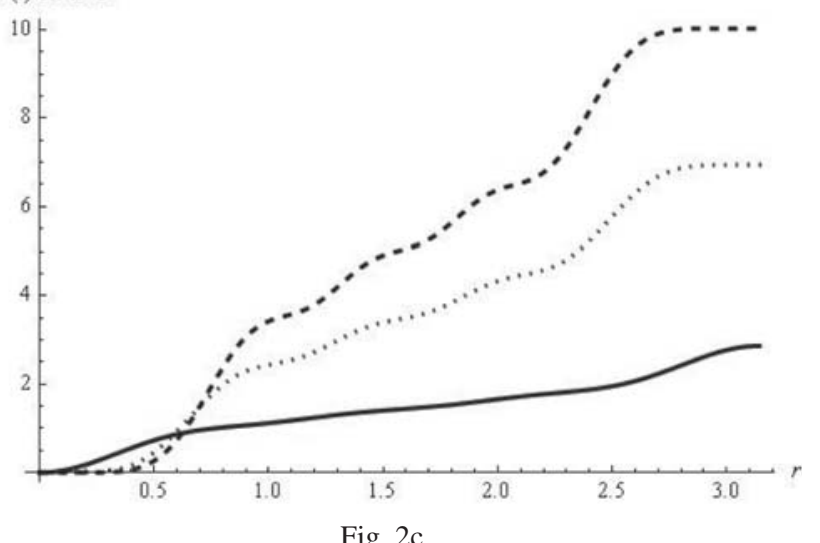

Fig. 2: Comoving particle number $\mathrm{Na}^{3}(\mathrm{r})$ within the volume of radius r. The solid, dotted and dashed curves are for $l=1 / 2,3 / 2$ and $5 / 2$ respectively. Figures a, b and c are for $\mathrm{n}=0,1$ and 2 respectively. In all the cases, the particle distributions seem to resemble that required for the flattened rotation curves of galaxies.

The comoving particle current is given by $\frac{d\left(\mathrm{Na}^{3}\right)}{d \eta}=\frac{R_{+}^{*} R_{-}}{2 i k} \frac{d T_{+}{ }^{2}}{d \eta}$.

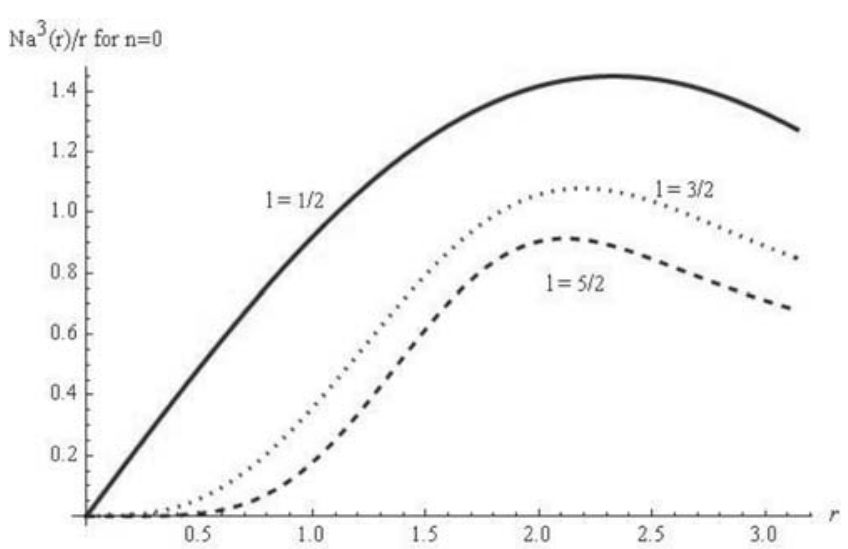

Fig. 3a: $\mathrm{Na}^{3} / \mathrm{r}$ for $\mathrm{n}=k-l-1=0$. The solid, dotted and dashed curves are for $\mathrm{l}=1 / 2,3 / 2$ and $5 / 2$ respectively. The quantity $\mathrm{Na}^{3} / \mathrm{r}$ is like the square of rotational velocity. So, as expected the curves are like rotation curves of galaxies.

$\mathrm{Na}^{3}(\mathrm{r}) / \mathrm{r}$ for $\mathrm{n}=1$

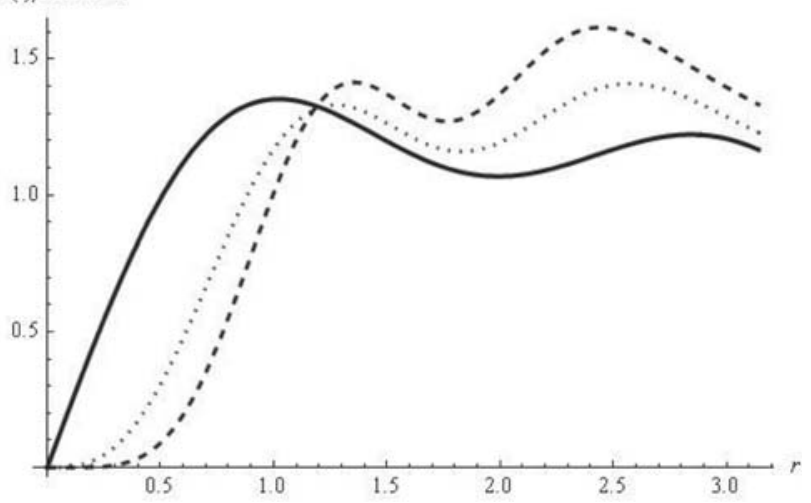

Fig. 3b: As in 3a for $n=1$.

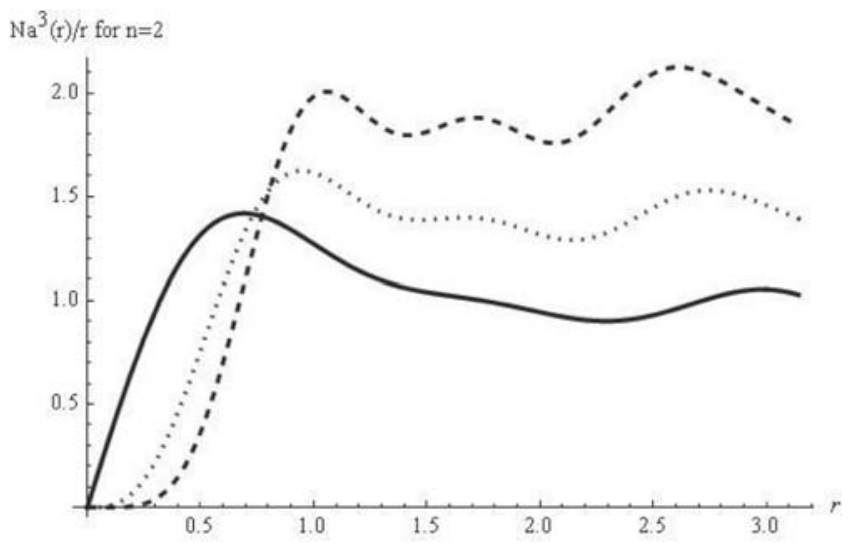

Fig. 3c: As in 3a for $n=2$.

Using equation (2), it simplifies to $\frac{d\left(N a^{3}\right)}{d \eta}=\frac{R_{+}^{*} R_{-}}{2}\left(T_{+}^{*} T_{-}-T_{+} T_{-}^{*}\right)$ which vanishes at $\eta_{0}$ showing conservation of the comoving particle number, otherwise it gives a net particle current. The solution of the time part and hence this current will be calculated in future works. 


\section{CONCLUSION}

Since all the matter particles are ultimately fermions, the study of Dirac field in the real universe is very important. In this paper, we have looked at the behavior of the particle number and particle current of the free Dirac field in the closed FRW universe. Although the particle number is conserved for the massless fields as expected, it is not for the massive ones. So, there is a finite particle current in any finite sized volume of the universe. Such currents tend to enhance the density contrast over time. It appears that the momenta of the Dirac particle is quantized in a closed FRW model, the quantization condition for the comoving momentum being $k=l+n+1$ where $n \geq 0$ is an integer, and the total angular momentum $l$ is a half-integer. As our Universe is almost flat, any overdensity region that has developed into structures should behave as a closed universe. Hence, Dirac particles that are gravitationally trapped in such structures will have their momentum quantized.

\section{REFERENCES}

[1] Newman, E.T. and Penrose, R. 1962. J. Math. Phys. 3: 566.

[2] Khanal, U. 2006. Class. Quantum Grav. 23: 4353.

[3] Chandrasekhar, S. 1983. The Mathematical Theory of Black Holes, Clarendon Press, Oxford).

[4] Khanal, U. 2006. ICTP preprint. IC/2006/136.

[5] Gradshteyn, I.S. and Ihzhik, I.M. 2000. Table of Integrals, Series, and Products. Sixth Edition. Academic Press. 